\title{
Einleitung: Interdisziplinäre Zugänge zu neuen Fragen des Urheberrechts
}

\author{
Hondros/Beaucamp/Fischer/Schrör
}

Der Stand des Urheberrechts im Jahr 2020 ist durch zwei Entwicklungen geprägt: Auf der einen Seite die Urheberrechtsreform der EU, mit der das Verhältnis von Internet-Plattformen, Verwertungsfirmen, Kreativen und Konsument:innen neu austariert und die bis zum Sommer 2021 in deutsches Recht umgesetzt werden soll; auf der anderen Seite der schon mehr als 20 Jahre währende „Metall auf Metall“-Urheberrechtsstreit um ein knapp zweisekündiges Sound-Sample, das ein Musikproduzent Ende der 1990er Jahre ohne Genehmigung der Rechteinhaber verwendet hatte. Beide Entwicklungen haben auf eigene Weise gesellschaftliche Konfliktlinien um urheberrechtliche Fragen freigelegt - und damit verbundene kulturelle, ökonomische, technologische, ethische und politische Ebenen sichtbar gemacht.

Im Fall der Urheberrechtsreform zeigt sich, wie stark die digitale Vernetzung durch das Internet die etablierten Kreativindustrien verändert hat und in die Lebenswelten der Menschen vorgedrungen ist. In den letzten 25 Jahren ist eine Generation von Menschen herangewachsen, für die der Zugriff auf digitale Medieninhalte wie Nachrichten, Videoclips oder Musik untrennbar zum Alltag gehört und die das Urheberrecht als politisch relevanten Gegenstand begreift. Und doch waren Größe und Lautstärke des Protests gegen die EU-Urheberrechtsreform überraschend, als im Frühling 2019 mehrere zehntausend junge Leute in Berlin gegen die befürchteten Einschränkungen „ihres“ Internets auf die Straße gingen. Was also hat dazu geführt, dass sich um Fragen des Urheberrechts derart viel politisches Momentum entwickeln konnte?

Um eine Antwort hierauf geben zu können, ist das Verhältnis von Technologie und Recht näher zu betrachten, das sich im Fall des Urheberrechts in den vergangenen zwei Dekaden grundlegend verändert hat. War das Urheberrecht bis vor einigen Jahrzehnten noch ein relativ spezielles Rechtsgebiet, das vor allem die Tätigkeiten von professionellen Kreativen, Verlagen und Technologieherstellern betraf, hat sich diese Zone in Richtung der Konsument:innen ausgeweitet, die dank digitaler Werkzeuge niedrigschwellig und intuitiv selbst zu Urheber:innen werden - aber auch genau- 
so schnell die Urheberrechte von Dritten verletzen können. Möglich ist das aufgrund digitaler Geräte und Software, aber auch aufgrund der digitalen Vernetzung, die das Internet und besonders Plattformen zum Austausch, zur Veröffentlichung und Verwertung von nutzergenerierten Inhalten, wie YouTube, Facebook oder TikTok bieten.

Natürlich gelten urheberrechtliche Regeln auch für Amateur:innen und Laien, die Remixe, Memes oder andere niedrigschwelle Bearbeitungen geschützter Werke erstellen und als nutzergenerierte Inhalte veröffentlichen. Es ist genauso naheliegend wie fair, dass Internetplattformen Urheber:innen an Einnahmen beteiligen, die sie beispielsweise über Werbemaßnahmen im Zusammenhang mit usergenerierten Inhalten verdienen. Dessen ungeachtet sind die dafür grundsätzlichen Regeln in Deutschland weiterhin ungeklärt, wie insbesondere der Fall um „Metall auf Metall“ klar macht: Nachdem der Popmusikproduzent Moses Pelham für einen Song ein knapp zweisekündiges Rhythmus-Sample aus dem Stück „Metall auf Metall“ von Kraftwerk verwendet hatte, entzündete sich ein bis heute anhaltender Streit um regulative Fragen der Remixkultur. Dieser Streit berührt auch grundlegende Fragen um Sinn und Funktion des Urheberrechts, etwa dessen Verhältnis zu Leistungsschutzrecht und Kunstfreiheit, den Auslegungsspielräumen bezüglich Schöpfungshöhe und freier Benutzung sowie der Beziehung von ästhetischer Referentialität und Werkkonkurrenz. In der Rückschau mag der 20 Jahre währende Streit um ein nicht einmal zwei Sekunden langes Rhythmus-Sample abstrakt und weit gegriffen wirken - werden die digitale Vernetzung und die Demokratisierung der Publikationsmittel durch das Internet als Faktoren mit einbezogen, so erhalten die genannten Punkte eine dringliche, ganz konkrete Relevanz, die weit über den Bereich der Popmusik hinausweist und zahlreiche Bereiche der Kulturproduktion und Alltagskommunikation betrifft.

Der vorliegende Sammelband widmet sich den geschilderten urheberrechtlichen Problemen und Phänomenen aus einer dezidiert interdisziplinären Perspektive. Vorbereitende Diskussionsprozesse hatten ihren Ausgang zunächst im Fachausschuss „Urheberrecht“ der Gesellschaft für Musikwirtschafts- und Musikkulturforschung (GMM) genommen. Diesem offenen Diskussionsraum gehören Musikwissenschaftler:innen, Sozialwissenschaftler:innen und Rechtswissenschaftler:innen aktiv an. Der Ausschuss ist seit seiner Gründung 2016 kontinuierlich gewachsen und unterstreicht damit das Interesse an einer Disziplinen vernetzenden Diskussion zum Urheberrecht. Aus diesem Wachstum entstanden auch Verbindungen zum Weizenbaum-Institut, dessen Ausrichtung auf die Wechselbeziehungen zwischen Digitalisierung und Gesellschaft nicht zuletzt urheberrechtliche Fragen adressiert. Im breiteren wissenschaftlichen Kontext stärken For- 
schungsprojekte wie das DFG-Projekt „Organizing Creativity under Regulatory Uncertainty: Challenges of Intellectual Property“ oder das im DFGGraduiertenkolleg "Innovationsgesellschaft heute” geförderte Projekt „Urheberrecht und Kreativität in der samplingbasierten Musikproduktion" der gegenwärtigen Formierung eines Forschungsschwerpunkts den Rücken, der sich neuen Fragen des Urheberrechts annimmt.

\section{Tipping Points}

Die Denkfigur des tipping points im Urheberrecht stellt das übergreifende Element aller Beiträge in diesem Band dar. Anhand dessen werden Grenzfragen des Urheberrechts untersucht. Tipping points verstehen wir als Wende- oder Kipppunkte, die Freiheit ermöglichen und/oder Restriktion bedingen, an denen etwas Neues entsteht oder ein Prozess eine andere Richtung einschlägt. Manche Arbeiten in diesem Band greifen die Denkfigur des tipping points zentral auf, bei anderen Beiträgen schwingt diese im Hintergrund mit. So leitet Heldt ihren Beitrag mit der Definition von Merriam-Webster zum tipping point ein. Bei einem tipping point handelt es sich hiernach um einen „kritischen Punkt in einer Situation, einem Prozess oder einem System, über den hinaus eine signifikante und oft unaufhaltsame Wirkung oder Veränderung stattfindet" ${ }^{\text {" }}$. Das Verständnis der Denkfigur, das den unterschiedlichen Beiträgen zugrunde gelegt wird, variiert durchaus. So wird diese teilweise als historischer Wendepunkt gesehen, als ein Ereignis, das im Nachhinein als Wendepunkt beschrieben werden kann, teilweise als Scheidepunkt künftiger Entwicklung, teilweise auch als Umbruchszeit, die Veränderungen bedingt, etwa in Bezug auf Akteurskonstellationen, Diskurse oder einzelne Praktiken.

Das unterschiedliche Verständnis der Denkfigur und der entsprechende Umgang damit ermöglichen, die Vielschichtigkeit der Dimensionen der Kipppunkte im Urheberrecht aufzuzeigen. Zentral war im Zusammenhang der Tagung, aus der die Beiträge entstanden sind, dass die jeweiligen Fragestellungen nicht nur aus einer Perspektive betrachtet werden, sondern aus verschiedenen Blickwinkeln ohne hierbei an disziplinären Grenzen Halt zu machen. Nur so können Kipppunkte erkannt, betrachtet und analysiert werden. Die Denkfigur der tipping points ermöglicht, Verände-

1 Definition von Merriam-Webster, freie Übersetzung von Amélie Heldt: „critical point in a situation, process, or system beyond which a significant and often unstoppable effect or change takes place". 
rungen, Entwicklungen und Unsicherheiten, denen das Urheberrecht unterliegt, in ihrer Dynamik einzufangen und entscheidende Aspekte dieser sehr fluiden Prozesse herauszuarbeiten. Dieser Vorgang wurde durch die intensive Diskussion im Rahmen der Tagung verfeinert, so dass jeder Beitrag für sich auf spezifische Art und Weise einen oder mehrere tipping points konturiert.

\section{Zu den Beiträgen}

Der Sammelband besteht aus insgesamt 13 Forschungsbeiträgen, die in drei thematisch abgegrenzte Teile gegliedert sind. Der erste Teil widmet sich dem Wandel der rechtlichen Rabmenbedingungen, dem Kreativität unterworfen ist und liefert anhand von fünf Fallstudien verschiedene Antworten. Den Auftakt macht Amélie Heldt: Sie untersucht anhand der EU-Reform des Urheberrechts auf Grundlage sozialwissenschaftlicher Theorien der Öffentlichkeit, welche tipping points sich im Gesetzgebungsverfahren identifizieren lassen. Dabei arbeitet sie insbesondere die Rolle der Öffentlichkeit in diesem heraus. Marion Goller schließt in ihrem Text an die EUReform an, und fokussiert aber auf die sogenannten „Uploadfilter“, die im Reformprozess im Zentrum der öffentlichen Diskussion standen. Die Autorin skizziert, welche kulturellen und technologischen Auswirkungen sich durch eine flächendeckende Einführung von Uploadfiltern einstellen könnten, insbesondere was die Inhalte der public domain und der digital gestützten Alltagskommunikation betrifft. Auch Hans-Christian Gräfe und Jonas Kunze interessieren sich für die über Plattformen stattfindende Alltagskommunikation: Sie analysieren die rechtlichen Anforderungen und Besonderheiten der chinesischen Social Media-Plattform TikTok, die in Europa an Beliebtheit gewinnt. TikTok basiert auf nutzergenerierten Inhalten, die fortlaufend remixed und weiterverbreitet werden, was die Plattform einerseits für junge Leute attraktiv macht, aber auch urheberrechtliche Herausforderungen mit sich bringt. Während das Urheberrecht und auch die Monetarisierung auf Tiktok vor allem auf der Verwertung von schöpferischen Leistungen aus der Vergangenheit beruht, drehen Crowdfunding-Plattformen dieses Prinzip gewissermaßen um, insofern sie Finanzierungen zukünftiger Projekte ermöglichen. Diese Perspektive nimmt Thomas Ernst ein. Er untersucht am Beispiel eines erfolgreich per Crowdfunding finanzierten Sachbuchs die Bedingungen und Konsequenzen, die sich aufgrund des Crowdfunding-Geschäftsmodells für Autorschafts- und Werkvorstellungen ergeben. Den Abschluss des ersten Teils macht Malte Zill. Auf Grundlage historischer Dokumente bespricht er die organisatori- 
schen Praktiken der GEMA in den 1930er und 1940er Jahren und die darauf erfolgten öffentlichen Reaktionen von Musiknutzenden. Dies vergleicht er mit einer Reform der Tarifstruktur der Verwertungsgesellschaft aus dem Jahr 2012. Damit stellt Zill die technisch-rechtlichen Entwicklungen des Immaterialgüterrechts in einen größeren historischen Zusammenhang und arbeitet so Parallelen und Unterschiede in der Urheberrechtsvertretung der Verwertungsgesellschaft heraus.

Im zweiten Teil widmet sich der Sammelband vertieft den künstlerischen Praktiken, die anhand verschiedener Parameter wie Schöpfungshöhe, Referentialität oder Werkqualität diskutiert werden. Zunächst untersuchen Daniel Müllensiefen und Klaus Frieler die Güte und Notwendigkeit des urheberrechtlichen Prinzips des sogenannten „starren Melodienschutzes", also der rechtlichen Privilegierung der Melodie. Dafür legen sie eine umfangreiche quantitative Analyse von melodischen Bausteinen zugrunde, anhand der sie zeigen, wie strukturelle Ähnlichkeit und das Kippen in neue, qualitativ eigenständig wahrgenommene Melodien zusammenhängen. Dario Haux greift in seinem Beitrag ebenfalls das Stichwort des tipping points auf, wenn er fragt, ob der Streit um das „Metall auf Metall"-Sample selbst als Wendepunkt in der Urheberrechtsdebatte verstanden werden kann. Zur Fundierung seiner Antwort zieht Haux das Urteil des Bundesverfassungsgerichts zu „Metall auf Metall“ heran, in dem er einige Ansatzpunkte eines raumsoziologisch gefärbten Verständnisses vom Internet als kreativ-alltäglichem Lebensraum erkennt. Thematisch schließt Konstantin Hondros an das Thema Sampling an, das einen Schwerpunkt in diesem zweiten Teil des Sammelbands darstellt. Hondros argumentiert noch stärker wirtschaftssoziologisch und tritt für ein Verständnis von Samples als flüchtigen Waren ein. Die besondere Qualität der Flüchtigkeit stellt er in seiner komparativen Analyse von Anbietern heraus, die auf den Handel von Samples spezialisiert und daher auch mit deren ökonomischmetrischen Herausforderungen der Vergleichbarkeit konfrontiert sind. Auch Sophie Beaucamp und Simon Schrör widmen sich dem Sampling: Sie verbinden eine systemtheoretisch informierte soziologische Perspektive auf das Verhältnis von Kunst, Wirtschaft und Recht mit einer juristischen Auseinandersetzung der rechtlichen Entwicklung im Sampling-Fall zu „Metall auf Metall“. Dabei identifizieren sie das Kriterium der Gewerblichkeit als tipping point und illustrieren an der Gruppe der Low-Budget-Musikproduzent:innen die feine Grenze, den Kipppunkt zwischen professioneller und hobbyhafter Musikproduktion. Christian Czychowski und Niclas Düstersiek greifen für ihren Beitrag den Themenschwerpunkt Sampling noch einmal auf. Unter Rückgriff auf die künstlerische Praxis der Appropriation Art und des damit verbundenen konzeptionellen Kunstbe- 
griffs im Sinne des Pastiches stellen sie eine Tradition zu modernen, digitalen und massenhaften Ausdrucksformen wie Sampling oder Remix her. Aus dieser Perspektive diskutieren die Autoren die verschiedenen Konsequenzen, die sich aus dem potentiellen Wegfall der urheberrechtlichen Schranke der freien Benutzung ergeben können.

Im dritten Teil des Sammelbands richtet sich der Blick in die Zukunft. Hier sind drei Beiträge zum Themenfeld Archivierung und Regulierung zusammengestellt, die die urheberrechtlichen Voraussetzungen und Implikationen für den Zugang zu Wissen, Daten und Sammlungen analysieren. Zunächst begibt sich Georg Fischer in den urheberrechtlich fragwürdigen bis sogar illegalen Bereich der wissenschaftlichen Schattenbibliotheken. Anhand verschiedener Beispiele, darunter SciHub oder UbuWeb, fragt der Autor, ob Schattenbibliotheken als Indikator für kippende Akteursgefüge in der akademischen Medienindustrie verstanden werden können, sich also ein tipping point in der Entwicklung des wissenschaftlichen Publizierens andeutet. Die technischen und rechtlichen Herausforderungen für Zugang und Archivierung sind auch das Thema von Miriam Akkermann. Am Beispiel der frühen Elektroakustischen Musik erläutert sie, wie Kompatibilität, Versionalität und Urheberschaft als musikalisch-technische Parameter bei der Archivierung berücksichtigt werden müssen und welche Probleme sich in diesem Feld aktuell und zukünftig stellen. Die noch grundsätzlichere, strukturelle Zugänglichkeit von materiellen und immateriellen Kulturgütern wird schließlich auch in dem gemeinsamen Beitrag von Fabian Rack, Franziska Boehm, Matthias Pasdzienery und Dörte Schmidt thematisiert. Sie stellen das Konsortium zur Nationalen Forschungsdateninfrastruktur von Kulturgütern (abgekürzt: NFDI4Culture) vor und skizzieren pragmatische Lösungsansätze, um unter Berücksichtigung der rechtlichen Voraussetzungen die vielfältigen Daten der Kulturlandschaft für die zukünftige Forschung verfügbar zu machen.

\section{Auf dem Weg zu einer interdisziplinären Urheberrechtsforschung}

Die Beiträge in dem vorliegenden Band weisen unter der Klammer der tipping points eine große Breite an theoretischen, methodischen und gegenständlichen Zugängen auf. Quantitative, musikpsychologische Untersuchungen, juristische Einordnungen, literaturwissenschaftliche Fallstudien oder soziologische Feldbeschreibungen zeigen, wie sehr das Urheberrecht die Wissenschaft umtreibt. Ganz gleich ob das Urheberrecht selbst Forschungsgegenstand ist, ob dessen Auswirkungen auf Forschungsobjekte untersucht werden, oder ob es um die Freiheiten und Restriktionen geht, 
die das Urheberrecht für das wissenschaftliche Handeln, das Forschen und Publizieren selber oder für das Archivieren hat: Akademisch finden sich viele unterschiedliche Herangehensweisen, die sich in Gänze einem Feld der Urheberrechtsforschung zuordnen lassen. Eine solche Feldzuordnung ist heuristisch natürlich interessant, reicht aber allein freilich nicht aus, um aktiv Denken über die eigene Disziplin hinaus zu erweitern. Hierzu bedarf es einer Verstetigung diverser, zum Teil bereits hier skizzierter Forschungsstrukturen und -haltungen, die eine interdisziplinäre Urheberrechtsforschung fördern und animieren, und zugleich den Qualitätsanforderungen innerhalb etablierter Fachcommunities und Wissenschaftszweige entsprechen.

Die Tipping Points Tagung 2020 verstand sich als Stimulus einer aktiven akademischen Vernetzung und gegenseitiger Einbeziehung, um das Feld der Urheberrechtsforschung um eine funktionale und produktive Form der Interdisziplinarität zu erweitern. Eine besondere Rolle kommt dabei den vor der Tagung erarbeiteten und allen Teilnehmenden zur Verfügung gestellten Workingpaper zu. Die beiden Konferenztage waren hierdurch ein interdisziplinäres Arbeitstreffen, auf dem die im Vorfeld ausgetauschten schriftlichen Beiträge kurz vorgestellt und umfassend diskutiert wurden. Die inhaltliche Breite der Beiträge und die Multidisziplinarität der Forschenden waren von vornherein bekannt. Die Autor:innen nutzten das Format der Tagung gezielt zur Diskussion von Fragen sowie zur Weiterentwicklung der Paper. Das bewusste Teilen der eigenen Expertise über die eigene Arbeit hinaus hat sich als vielleicht wichtigster Aspekt des gemeinsamen Projektes herausgestellt. Leitlinie der Zusammenarbeit und des Austausches aller Autor:innen in diesem Band war und ist es, das eigene Wissen über urheberrechtlich relevante Bereiche weiterzutragen und das gegenseitige Informieren als Modus Operandi zu verfolgen. Dies erfordert nicht nur eine grundsätzliche Offenheit gegenüber Interdisziplinarität, sondern auch eine kritische Grundhaltung gegenüber Prämissen der eigenen Disziplin.

Nun ist und bleibt interdisziplinäre Forschung stets ein Wagnis. Die Gefahren einer Profanisierung von Erkenntnissen oder dem Einigen am kleinsten gemeinsamen Nenner bestehen ebenso wie die Sorge vor fehlender Anschlussfähigkeit zu disziplinären Fachdiskursen. Eine interdisziplinäre Forschungshaltung sollte mit einem Bewusstsein gegenüber diesen Gefahren und Sorgen einhergehen und den (Mehr)Wert des Interdisziplinären kritisch hinterfragen. Wie sich in diesem Band zeigt, kann Interdisziplinarität ganz unterschiedliche Ausformungen haben. Sie fängt an bei dem Wissen um die Argumente und Sichtweisen anderer Disziplinen auf den gleichen Gegenstand und führt über eine Verzahnung unterschiedli- 
cher Forschungs- und Erkenntnislogiken hin zu genuin supradisziplinären Fragestellungen und der Entwicklung neuer Forschungsideen, die Fragen stellen, die monodisziplinär unbeantwortbar wären. Diese Vielfalt gelebter Interdisziplinarität anzuerkennen und als Stärke zu begreifen, ist eines der Anliegen des Buches.

Die Breite der hier versammelten Themen und Herangehensweisen illustriert, wie groß die Relevanz ist, die das Urheberrecht in einer vernetzten Gesellschaft hat, und dass das Urheberrecht ein Zukunftsthema ist und bleibt: Das gilt innerhalb der Wissenschaft, beim Umgang mit den immer umfassender vorliegenden Materialien, die archiviert, katalogisiert und zugänglich gemacht werden wollen, sowie bei Gedächtnisinstitutionen, die sich ähnlichen Herausforderungen stellen. Zugleich befinden wir uns mitten in der digitalen Transformation der Medienindustrie, der Unterhaltungsbranche, der Musik und der Literatur. All diese Entwicklungen und Umbrüche lassen sich nicht unabhängig vom Urheberrecht betrachten, sind aber zugleich viel mehr als juristische Problemstellungen, die monodisziplinär zu greifen oder gar zu lösen wären. Auch deswegen versteht sich dieser Band als Versuch, interdisziplinäre Zugangsmöglichkeiten zu illustrieren, die auch in Zukunft Anwendung auf die spannenden und vielfältigen Fragen des Urheberrechts in der vernetzten Gesellschaft finden können.

Auf der Tagung ist es gelungen, die Idee der Vernetzung und Verzahnung in den hier vorliegenden Beiträgen weiterzuführen und damit einen Schritt in Richtung einer lebendigen, interdisziplinären Urheberrechtsforschung zu machen. Eine solche Urheberrechtsforschung, die systematisch informiert von den Erkenntnissen und Erfordernissen anderer funktioniert und mitunter in konkreter Zusammenarbeit mündet, hat das Potenzial, die diversen wichtigen und aktuellen Forschungszweige in den Rechts-, Sozial-, Musik-, Literatur- und Kulturwissenschaften um eine integrierende Perspektive zu erweitern. 


\section{Danksagung}

Unser Dank dafür, diese Arbeit vorlegen zu können, gebührt vor allem den Autor:innen des Bandes und den Teilnehmenden der Arbeitstagung, die gezeigt haben, dass ein intrinsisches Interesse und eine genuine Neugierde für interdisziplinäre Ansätze der Forschung zum Urheberrecht besteht. Wir danken zudem den Kolleg:innen in der GMM, besonders Holger Schwetter und Frédéric Döhl, die die Tagung mit uns vorbereitet, angedacht und durchgeführt haben, sowie den Reviewer:innen für ihre kritische Arbeit. Herzlichen Dank auch an Axel Metzger für das große Vertrauen in dieses Projekt. Ebenso danken wir Jana Pinheiro, Alexandra Keiner, Valeria Nieberg und Huy Nguyen für die Unterstützung an vielen kleinen und großen Stellen.

Unser Dank gilt schließlich dem Bundesministerium für Bildung und Forschung (BMBF) dessen Förderung des Weizenbaum-Instituts für die vernetzte Gesellschaft (Förderkennzeichen: 16DII111 - „Deutsches Internet-Institut") unter anderem die Open Access-Veröffentlichung dieses Bandes möglich gemacht hat. 
